\title{
Additional Transition Line in Jammed Asymmetric Bidisperse Granular Packings
}

\author{
Juan C. Petit $\odot,{ }^{1}$ Nishant Kumar, ${ }^{1}$ Stefan Luding, ${ }^{2}$ and Matthias Sperl $\odot^{1,3}$ \\ ${ }^{1}$ Institut für Materialphysik im Weltraum, Deutsches Zentrum für Luft- und Raumfahrt (DLR), 51170 Köln, Germany \\ ${ }^{2}$ Multi-Scale Mechanics (MSM), Faculty of Engineering Technology, MESA+, University of Twente, \\ PO Box 217, 7500 AE Enschede, The Netherlands \\ ${ }^{3}$ Institut für Theoretische Physik, Universität zu Köln, 50937 Köln, Germany
}

(Received 26 January 2020; revised 8 July 2020; accepted 7 October 2020; published 17 November 2020)

\begin{abstract}
We present numerical evidence for an additional discontinuous transition, upon compression, inside the jammed regime for an asymmetric bidisperse granular packing. This additional transition line separates jammed states with networks of predominantly large particles from jammed networks formed by both large and small particles, and the transition is indicated by a discontinuity in the number of particles contributing to the jammed network. The additional transition line emerges from the curves of jamming transitions and terminates in an end point where the discontinuity vanishes. The additional line is starting at a size ratio around $\delta=0.22$ and grows longer for smaller $\delta$. For $\delta \rightarrow 0$, the additional transition line approaches a limit that can be derived analytically. The observed jamming scenarios are reminiscent of glass-glass transitions found in colloidal glasses.
\end{abstract}

DOI: 10.1103/PhysRevLett.125.215501

The jamming transition at packing fraction $\phi_{J}$ in granular matter is indicated by a discontinuous jump in the number of contacts per particle [1-6]. For frictionless monodisperse packings, that value is close to $\phi_{J} \approx 0.64$ in $3 \mathrm{D}[5,7]$. To suppress crystallization, bidisperse packings are introduced where $\phi_{J}$ can be tuned to higher values by varying the size ratio $\delta$ and volume concentration of small particles $X_{S}$ [8-12]. Such mixtures are also relevant for industrial processes since mechanical properties of bidisperse packings such as bulk modulus and wave speed can be controlled [13].

While the dependence of the jamming density on $\delta$ and $X_{S}$ has been studied previously [8-12], a better understanding of jammed states in highly asymmetric bidisperse mixtures for extremely low $X_{S}$ is intended here. For example, previous works have assumed that the jammed structure of a bidisperse packing is formed by the equal contribution of both large and small particles since both species jam simultaneously at $\phi_{J}$ [9-11]. This is true above certain values of $\delta$ and $X_{S}$, since then the particle sizes are similar enough that both species follow the same behavior. However, for lower values of $\delta$ and $X_{S}$, each component behaves differently when approaching jamming, which suggests a potential decoupling in the jamming in the binary system. Indeed, it has been recently demonstrated in Ref. [8] that the jammed structure of an asymmetric

Published by the American Physical Society under the terms of the Creative Commons Attribution 4.0 International license. Further distribution of this work must maintain attribution to the author(s) and the published article's title, journal citation, and DOI. bidisperse system evolves from a small-sphere rich to a small-sphere poor structure. Such behavior is marked by an abrupt drop in the number of small particles contributing to the jammed structure at a specific $X_{S}$, while the rest of the small particles remain without contacts.

The recent works cited above indicate that, in principle, two different pathways to jamming need to be distinguished for small $\delta$ : one driven by predominantly large particles and one driven by both species together. It is the aim of this Letter to demonstrate explicitly that such distinction can be made rigorous by an analysis of the partial contact numbers between particles of different species. This leads to the identification of an additional transition between different jammed states for $X_{S}<X_{S}^{*}(\delta)$ for $\delta \leq \delta^{*}$. The transition diagram for that scenario is shown in Fig. 1 and shall be elaborated on below. In the following, the simulation method shall be explained, followed by the analysis and discussion of the results; the results are rationalized theoretically and put into context with scenarios found in glasses in the Conclusion.

Simulations using the 3D discrete element method are performed with the software MercuryDPM [13,14]. Bidisperse packings are formed by $N=6000$ particles, where a number of large $N_{L}$ and small $N_{S}$ particles with radius $r_{L}$ and $r_{S}$ are considered, respectively. Each packing is characterized by the size ratio, $\delta=r_{S} / r_{L}$, and the concentration of small particles, $X_{S}=N_{S} \delta^{3} /\left(N_{L}+N_{S} \delta^{3}\right)$, as well as the overall packing fraction $\phi$ (see Supplemental Material, Sec. I for further details [15]). We restrict ourselves to the isotropic deformation and to the linear contact model without any friction between particles [16,17]. Thus, we exclude all the nonlinearities present in the system due to contact models 


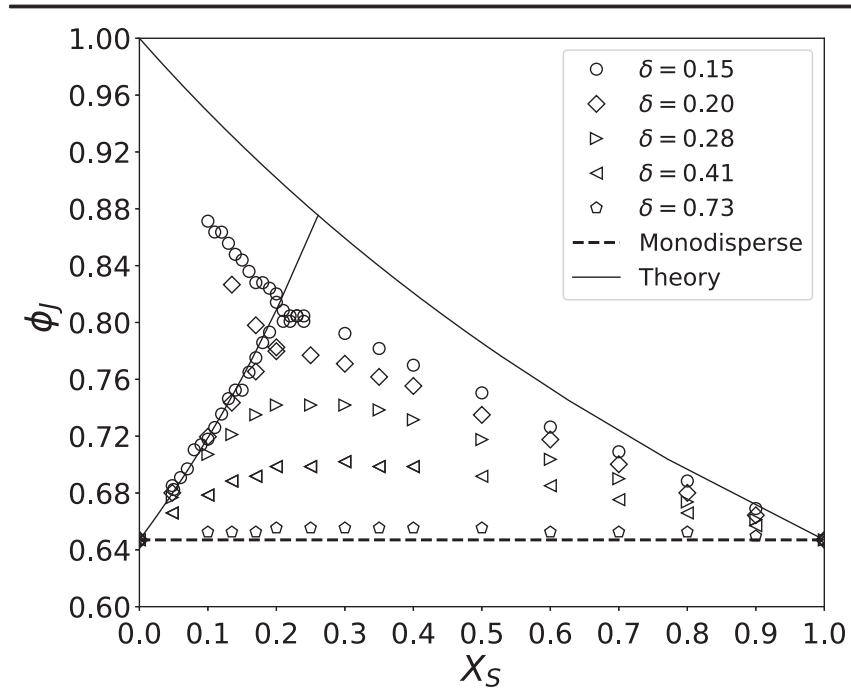

FIG. 1. Jamming density $\phi_{J}$ as a function of the volume concentration of small particles $X_{S}$ for different values of the size ratio $\delta$. The extreme $X_{S}$ values (zero and one) correspond to monodisperse systems where $\phi_{J} \approx 0.645$ indicated by the dashed horizontal line. Solid lines represent the result of Eq. (1).

and analyze the effect of the size ratio and concentration of small particles at jamming. Each bidisperse packing characterized by the parameters $\left(\delta, X_{S}\right)$ is created and further compressed using an unique, well-defined protocol $[16,18]$. $\phi_{J}$ for each configuration is determined at the point where the partial mean contact number exhibits a sharp decay. The values of $\phi_{J}$ as a function of $X_{S}$ for different $\delta$ are shown in Fig. 1.

For a particular $\delta=0.15$, the jamming density increases with $X_{S}$ up to a maximum value occurring at $X_{S}^{*}(0.15) \approx$ 0.21 , then decreases for larger values. Along the increasing transition line, jamming is driven by the force network created by the large particles, since most of the small ones remain without or with only few contacts in the cages formed by the large ones. This is confirmed when looking at Figs. 2(a) and 2(c), where mainly large particles are carrying the load in the jammed state. On the other hand, on the decreasing branch of $\phi_{J}$ for high $X_{S}$, small and large particles arrest simultaneously at the same density, see Figs. 2(b) and 2(d). However, for lower values of $\delta$ and $X_{S}$, a decoupling in the mean contact number $Z$ between large and small particles is observed, see Fig. 2(a). Large particles arrest first at lower $\phi$, while small ones remain without contacts. Making the packing denser, small particles exhibit an apparent jump in $Z_{S S}$ and $Z_{\text {mix }}$ [see Fig. 2(a)], indicating that a fraction of them start contributing in a discontinuous fashion to the already jammed structure of large ones. While the related conditions are certainly fulfilled by the setup of the simulation, simple Maxwell counting arguments or variations thereof [19] are not sufficient to explain the complex variation of contact numbers in that regime.

To corroborate the behavior observed in the $Z_{S S}$, in addition we have quantified the fraction of large $n_{L}$ and
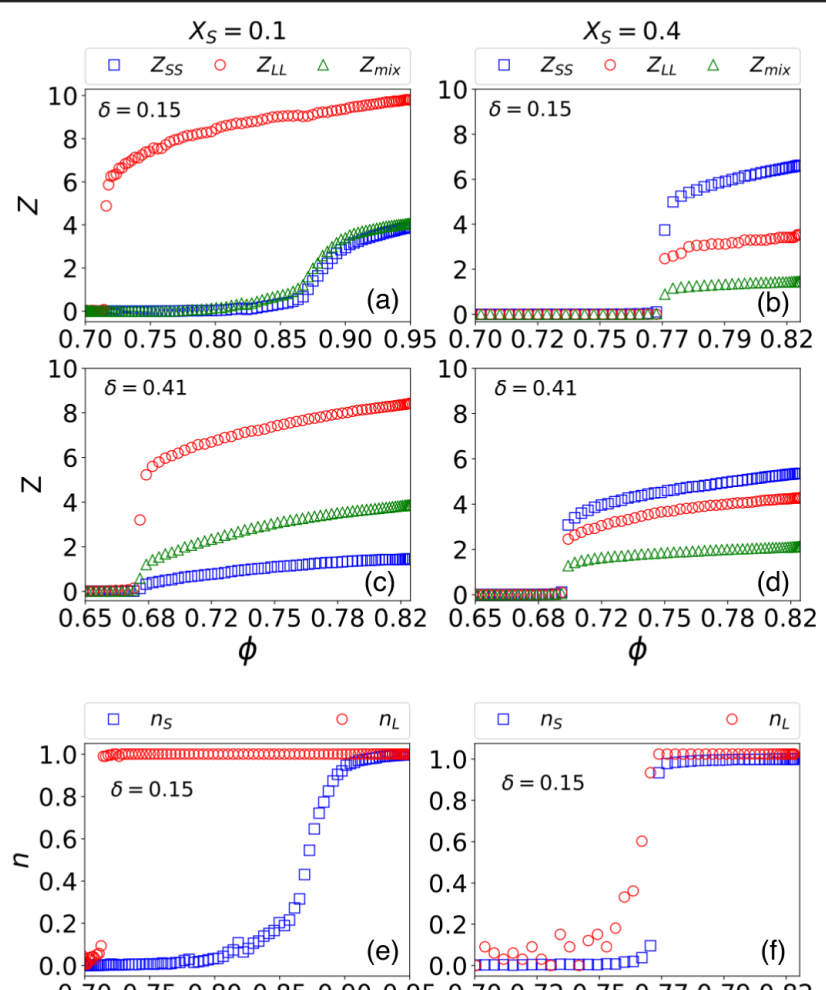

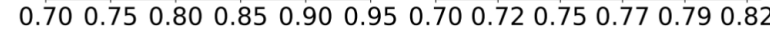

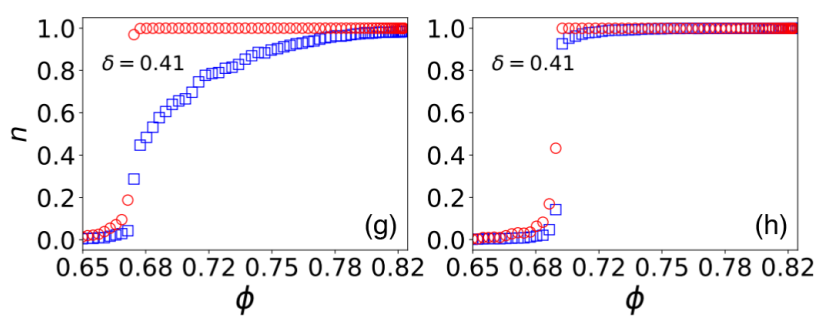

FIG. 2. (a)-(d) Mean contact number $Z$ for each type of contact as a function of $\phi$ for four combinations of $\delta$ and $X_{S} . Z_{n m}$ corresponds to the sum of contacts between particles of species $n$ and $m$ divided by total number of $N$ particles, with $n, m \in[L, S]$. The analyzed data are taken along the decompression branch. $Z_{\text {mix }}$ is defined as $Z_{\text {mix }}=\left(Z_{L S}+Z_{S L}\right) / N$. (e)-(h) Fractions of large $n_{L}$ and small $n_{S}$ particles contributing to the force network as a function of the packing fraction $\phi$ for two sets of $\left(\delta, X_{S}\right)$.

small particles $n_{S}$ contributing to the force network as a function of $\phi$ shown in Figs. 2(e)-2(h). This quantity exhibits the additional jump more drastically compared to $Z$. A clear decoupling between $n_{L}$ and $n_{S}$ at lower $\delta$ and $X_{S}$ can be seen in Fig. 2(e), while for higher values of $\delta$, both sizes of particles contribute simultaneously to the jammed structure. Such decoupling indicates that a large amount of small particles are jammed discontinuously at higher densities, which resembles a similar behavior as by the large particles at low densities. To extract the precise value of the jamming density where $n_{S}$ jumps for low $X_{S}$, we used the derivative of $\partial n_{S} / \partial \phi$ (see Supplemental Material [15], Sec. II). Figure 1 displays such values for $X_{S}<X_{S}^{*}(\delta) \approx 0.21$, indicated by the increasing line of densities for smaller $X_{S}$. Such a line extends the transitions 


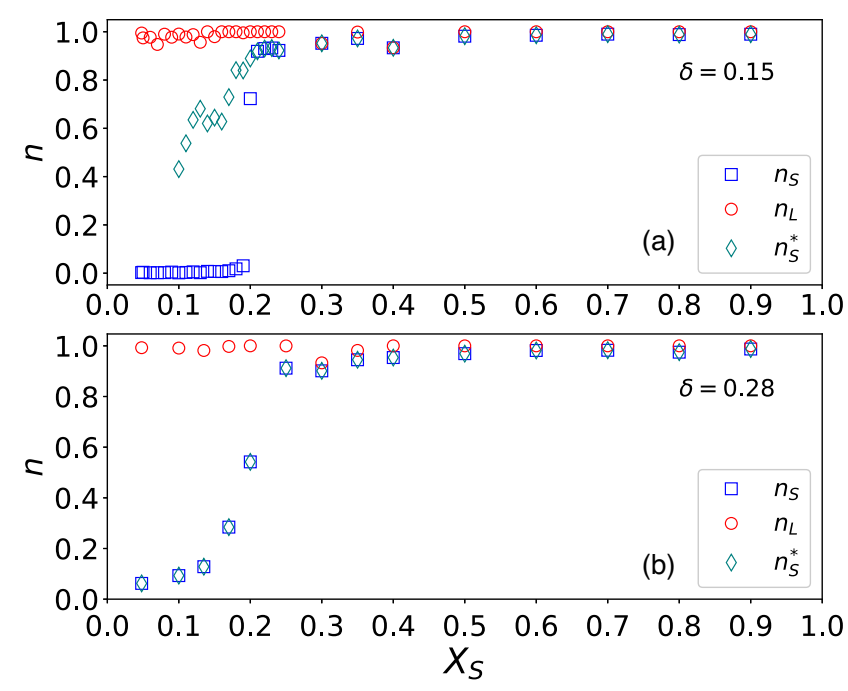

FIG. 3. Fraction of small $n_{S}$ and large particles $n_{L}$ contributing to the jammed structure as a function of the concentration $X_{S}$ of small particles for (a) $\delta=0.15$ and (b) $\delta=0.28$. $n_{S}^{*}$ represents the fraction of small particles along the additional line.

where both particle species are jammed into a regime of smaller $X_{S}$. For the larger $\delta=0.20$, the additional line emerges from $X_{S}^{*}(0.20) \approx 0.18$. For $\delta=0.15$ and 0.20 , the packing fractions where the additional transition lines emerge are $\phi^{*}=0.81$ and 0.79 , respectively.

Except for the additional transition line, the results shown in Fig. 1 are quite similar to those previously found in experiments using glass beads [11] and in 3D simulations $[9,10]$. In those latter works, however, no additional line was identified. Recent work [8], which has studied the statistics of the small particles in the jammed structure of bidisperse systems in more detail, showed that indeed the fraction of small particles contributing to the jammed structure decays to small values for $X_{S}<X_{S}^{*}$, which becomes discontinuous as $\delta$ decreases. This allows one to make a distinction between two regimes: one where small particles contribute to the jammed network jointly with large ones $\left(X_{S} \geq X_{S}^{*}\right)$ and the second one where only large particles contribute $\left(X_{S}<X_{S}^{*}\right)$. We have found that, besides these two regimes, there is an additional line emerging at $X_{S}=X_{S}^{*}$. The additional line represents a jammed state $\phi_{J}$, extending into higher densities and terminating in an end point $\left(X_{S}^{\circ}(\delta), \phi^{\circ}(\delta)\right)$. The additional line starts emerging for $\delta<\delta^{*}=0.22$ where the line's end point extends further to lower $X_{S}$ as $\delta$ decreases.

The evolution of the fractions of small and large particles that contribute to the jammed structure, $n_{S}$ and $n_{L}$, along the jamming lines elucidates the different nature of the transitions, i.e., by discussing the jump heights in these quantities as the jamming transition is crossed. These ratios are shown in Fig. 3 for two representative values of $\delta$. Here, we extracted $n_{S}$ and $n_{L}$ from the points where the largeparticle contribution jumps and $n_{S}^{*}$ from those where the small-particle contribution jumps. The existence of the additional transition line is now manifested in the cases where $n_{S}^{*}$ splits from $n_{S}$, see Fig. 3(a) at low $X_{S}$. Close to the crossing point where the additional transition line emerges, the difference between $n_{S}$ and $n_{S}^{*}$ is largest. For lower $X_{S}$, the second jump $n_{S}^{*}$ has to be compared with the value $n_{S}$ that is increasing regularly with packing fraction after the first transition: once $n_{S}$ and $n_{S}^{*}$ become of equal value, no more second jump can be identified, the end point of the additional transition line has been reached.

Technically, such a cessation of a second jump is more difficult to identify precisely than a clearly developed second jump close to the crossing point, which explains the fluctuations in Fig. 3. The sharp rise in $n_{S}$ around $X_{S}^{*} \approx 0.21$ has been noted before [8]. Within the results obtained here, it can be seen as a natural consequence from the crossing of a line with a finite jump in $n_{L}$ and a line with a finite jump in $n_{S}^{*}$. For $\delta=0.28$ [Fig. 3(b)], $n_{S}^{*}$ and $n_{S}$ merged into one line at lower $X_{S}$, suggesting that no additional transition line is found (see Fig. 1).

A comprehensive view of the evolution of the configurations of force-carrying particles including the history of the established contacts is shown in Fig. 4 for $\delta=$ 0.15 and $X_{S}=0.1$, as well as in the Supplemental Material videos [15] for $\delta=0.15$ and $X_{S}=0.1,0.15,0.19$, and 0.21, respectively. In the left column of Fig. 4 , the contact-bearing large particles are shown from $\phi=0.72$ at the first transition to $\phi=0.871$ and $\phi=0.875$, which is slightly before and after the second transition. Little apparent changes are visible here. In contrast for the small particles, at the first transition shown in the top row of Fig. 4, only very few of the small particles are arrested by permanent contacts. Between $\phi=0.72$ and $\phi=0.871$, gradually more of the small particles also establish contacts and contribute to the force network. At $\phi=0.875$ another discontinuous jump is seen, with many of the small particles becoming arrested all at once rather than gradually.

Figure 4 shows the second discontinuity well separated from the first one, as the point $\delta=0.15$ and $X_{S}=0.1$ is already far inside the jammed regime and hence comparably far away from the first line. At the same time, the point is close to the end point where the second discontinuity vanishes, and hence the discontinuity shown in Fig. 4 is rather more moderate. More drastic jumps can be seen when getting closer to the crossing at $X_{S}^{*}=0.21$, where, in turn, the location is closer to the first line. The videos in the Supplemental Material [15], showing the evolution of the packing with increasing density along the additional line, clearly indicate different discontinuities.

The additional transition lines are a result of the decoupling of the jamming transition between large and small particles for low $X_{S}$ and low $\delta$. Such a scenario was introduced by Furnas almost a century ago [20] to predict the highest density of aggregates entering in the manufacture of mortar and concrete. This model can be evaluated 

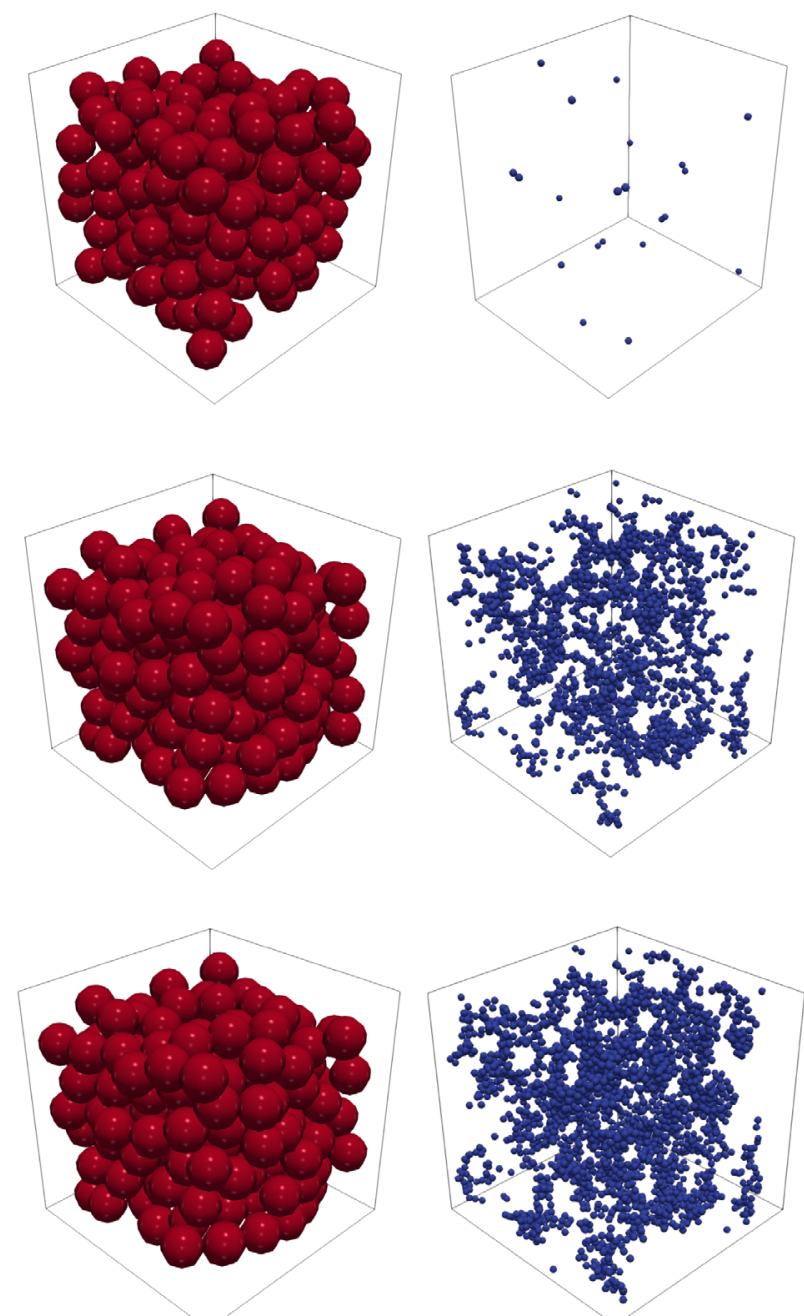

FIG. 4. Evolution of the configuration of force-carrying large (left column) and small (right column) particles for $\delta=0.15$ and $X_{S}=0.1$. The top row shows results at the transition of a loose assembly to a first force-carrying packing at $\phi=0.72$. The middle row is shown for $\phi=0.871$ just before the additional transition line, and the bottom row exhibits the results and configuration for the additional transition line at $\phi=0.875$.

analytically if the size ratio of the particles is in the extreme limit $\delta \rightarrow 0$. Then, $\phi_{J}$ can be written as two lines sharing a common point at $\hat{X}_{S}^{*}$, with $\phi_{\text {rcp }}$ designating the packing fraction at random close packing $(\mathrm{RCP})$

$$
\lim _{\delta \rightarrow 0} \phi_{J}\left(X_{S}\right)=\left\{\begin{array}{l}
\phi_{\mathrm{RCP}} /\left(1-X_{S}\right), \\
\phi_{\mathrm{RCP}} /\left[\phi_{\mathrm{RCP}}+\left(1-\phi_{\mathrm{RCP}}\right) X_{S}\right],
\end{array}\right.
$$

where the upper line is usually considered for $0 \leq X_{S}<\hat{X}_{S}^{*}$, while the lower line is deemed applicable for $\hat{X}_{S}^{*} \leq X_{S} \leq 1$. The other branches are usually discarded. Looking at the trend in Fig. 1 for $\delta \rightarrow 0$, one may extrapolate the simulation results to the lines given in Eq. (1). In particular, the lower expression in Eq. (1) may in fact may be applicable for the whole range $0 \leq X_{S} \leq 1$.

Equation (1) predicts a line crossing with a packing fraction of $\phi_{J}\left(\hat{X}_{S}^{*}\right)=0.87$ at $\hat{X}_{S}^{*}=\left(1-\phi_{\mathrm{RCP}}\right) /\left(2-\phi_{\mathrm{RCP}}\right)=$ 0.26 , which is in reasonable agreement with the values obtained here for $X_{S}^{*}$ at finite $\delta$, especially when observing the upward trend in $X_{S}^{*}$ from the simulations (cf. Fig. 1). The Furnas model then predicts an additional transition line ending at a density of unity. In Fig. 1, the additional line given by the simulation data follows qualitatively that line and ends at progressively lower values $X_{S}^{\circ}(\delta)$ for smaller $\delta$. One may hence assume-without further proof at this point - that the extension of the Furnas formula provides a reasonable estimate for the limit of a spacefilling packing within the peculiar limit where the volume of each individual small sphere vanishes with $\delta \rightarrow 0$, but where at the same time the volume contribution of the small particles remains finite for each $X_{S}>0$. In the simulation for finite $\delta$, the transition line ends at $X_{S}^{\circ}>0$ and no discontinuity is found in $n_{S}$ and $Z_{S S}$ for $X_{S}<X_{S}^{\circ}$ (see Supplemental Material [15], Sec. III).

In conclusion, we have shown that the jamming diagram in bidisperse packings is enriched by an additional transition. This transition appears for $X_{S}<X_{S}^{*}(\delta)$ when small particles get in contact with the jammed structure already formed by large particles. The trend in the evolution of the additional line lends support to the equations of the Furnas model, which predicts a similar additional line for the intricate limit of a space-filling binary packing. The results have been obtained using a specific simulation method described above and in more detail in the Supplemental Material [15], which includes, in addition, Refs. [3,21-35], and it remains open to what extent other protocols can exhibit the same scenarios. A physical interpretation is given in order to support the reliability of the results beyond a specific protocol or implementation. Also, most notably in the simulation work in Ref. [8], parts of the findings presented here are found as well, with the exception of an additional transition line. Within a different simulation protocol and data analysis, indications of an additional transition line have been found for a mixture with $\delta=0.17$ [35]. In addition to other computer simulations, however, the findings above should be well within the scope of experiments, e.g., along the lines of the original identification of the square-root bifurcation at jamming in two dimensions [6].

The results presented in Fig. 1 demonstrate an interesting connection to the glass-glass transition phenomenology in colloidal glasses in general [36] and binary colloidal suspensions in particular [37], thereby pointing toward a more close relationship between glass- and jammingtransition phenomena [38]: While glass and jamming transitions for hard spheres occur at different packing fractions 0.58 and 0.64 in experiments in three dimensions, they may well be considered more closely related in higher dimensions, thus allowing for a more unified description in 
the future. The consideration of multiple glass as well as multiple jammed states offers the opportunity to confront theoretical concepts with richer scenarios than the correspondingly more simple transitions. Also, the relevance of different length scales in the structure of packings [39] may well reveal additional features when more than one jammed state is considered.

The extension of the jamming line found here gives a richer interpretation of the subjamming transition, a term introduced in Ref. [8]. There, the crossing point $\left(\delta^{*}, X_{S}^{*}\right)$ has been interpreted as showing the hallmarks of a critical point akin to those of phase transitions. From our results here we interpret this point as rather a crossing point of two bifurcation lines, forming part of a more general bifurcation diagram. Two jamming lines cross here, and while one terminates at the crossing, the other one can continue until its end point. This additional transition line delineates a region where small particles are inside the interstices of an already jammed structure of large particles. Increasing $\delta$, the additional transition line shrinks, so that at some $\delta^{*}$, end point and crossing point coincide, $X_{S}^{\circ}\left(\delta^{*}\right)=X_{S}^{*}\left(\delta^{*}\right)$. The scenario of Fig. 1 hence allows for the interpretation within the framework of bifurcation theory, cf. [40], where the additional line with end point can be seen as a cusp bifurcation and the whole scenario with a vanishing cusp at some point a swallowtail singularity. Also, while the existence of these bifurcations can be considered a topologically robust phenomenon, the additional line may not necessarily emerge at the maximum in packing fraction for the respective jamming-transition curves. The outline of such scenarios based on the results above should stimulate developments in theory where the analogy with phase transitions as in Refs. [8,35] on one hand and bifurcations on the other present alternative pictures that should have consequences for theoretical predictions in the field.

We thank T. Kranz, P. Born, L. Elizondo-Aguilera, and Th. Voigtmann for helpful discussions about the results. This work was supported by the German Academic Exchange Service (DAAD). We also acknowledge partial funding from BMWi/DLR through Project No. 50WM2071.

[1] H. P. Zhang and H. A. Makse, Phys. Rev. E 72, 011301 (2005).

[2] E. Aharonov and D. Sparks, Phys. Rev. E 60, 6890 (1999).

[3] M. van Hecke, J. Phys. Condens. Matter 22, 033101 (2010).

[4] R. P. Behringer and B. Chakraborty, Rep. Prog. Phys. 82, 012601 (2019).

[5] C.S. O'Hern, L. E. Silbert, A. J. Liu, and S. R. Nagel, Phys. Rev. E 68, 011306 (2003).

[6] T. S. Majmudar, M. Sperl, S. Luding, and R. P. Behringer, Phys. Rev. Lett. 98, 058001 (2007).
[7] A. Donev, S. Torquato, F. H. Stillinger, and R. Connelly, J. Appl. Phys. 95, 989 (2004).

[8] I. Prasad, C. Santangelo, and G. Grason, Phys. Rev. E 96, 052905 (2017).

[9] A. B. Hopkins, Y. Jiao, F. H. Stillinger, and S. Torquato, Phys. Rev. Lett. 107, 125501 (2011).

[10] I. Biazzo, F. Caltagirone, G. Parisi, and F. Zamponi, Phys. Rev. Lett. 102, 195701 (2009).

[11] S. Pillitteri, G. Lumay, E. Opsomer, and N. Vandewalle, Sci. Rep. 9, 7281 (2019).

[12] N. Kumar and S. Luding, Granular Matter 18, 58 (2016).

[13] N. Kumar, V. Magnanimo, M. Ramaioli, and S. Luding, Powder Technol. 293, 94 (2016).

[14] P. A. Cundall and O. D. L. Strack, Géotechnique 29, 47 (1979).

[15] See Supplemental Material at http://link.aps.org/ supplemental/10.1103/PhysRevLett.125.215501 for details of the simulation algorithms, data analysis, and movies.

[16] O. I. Imole, N. Kumar, V. Magnanimo, and S. Luding, KONA Powder Part. J. 30, 84 (2013).

[17] N. Kumar, S. Luding, and V. Magnanimo, Acta Mech. 225, 2319 (2014).

[18] F. Göncü, O. Durán, and S. Luding, C. R. Mécanique 338, 570 (2010).

[19] P. K. Morse and E. I. Corwin, Phys. Rev. Lett. 119, 118003 (2017).

[20] C. Furnas, Ind. Eng. Chem. Res. 23, 1052 (1931).

[21] S. Luding, Powder Technol. 158, 45 (2005).

[22] N. P. Kruyt, I. Agnolin, S. Luding, and L. Rothenburg, J. Mech. Phys. Solids 58, 1286 (2010).

[23] O. Durán, N. P. Kruyt, and S. Luding, Int. J. Solids Struct. 47, 2234 (2010).

[24] J. Sun and S. Sundaresan, J. Fluid Mech. 682, 590 (2011).

[25] F. Alonso-Marroquin, S. Luding, H. J. Herrmann, and I. Vardoulakis, Phys. Rev. E 71, 051304 (2005).

[26] C. Thornton, Particuology 8, 119 (2010).

[27] C. Thornton and L. Zhang, Géotechnique 60, 333 (2010).

[28] A. Thornton, T. Weinhart, S. Luding, and O. Bokhove, Int. J. Mod. Phys. C 23, 1240014 (2012).

[29] T. Weinhart, A. R. Thornton, S. Luding, and O. Bokhove, Granular Matter 14, 289 (2012).

[30] C. S. O'Hern, L. E. Silbert, A. J. Liu, and S. R. Nagel, Phys. Rev. E 68, 011306 (2003).

[31] D. Koeze, D. Vågberg, B. Tjoa, and B. Tighe, Europhys. Lett. 113, 54001 (2016).

[32] S. Luding, Granular Matter 10, 235 (2008).

[33] C. S. O'Hern, S. A. Langer, A. J. Liu, and S. R. Nagel, Phys. Rev. Lett. 88, 075507 (2002).

[34] H. A. Makse, D. L. Johnson, and L. M. Schwartz, Phys. Rev. Lett. 84, 4160 (2000).

[35] Y. Hara, H. Mizuno, and A. Ikeda, arXiv:2009.01563.

[36] M. Sperl, Phys. Rev. E 69, 011401 (2004).

[37] T. Voigtmann, Europhys. Lett. 96, 36006 (2011).

[38] P. Charbonneau, J. Kurchan, G. Parisi, P. Urbani, and F. Zamponi, Annu. Rev. Condens. Matter Phys. 8, 265 (2017).

[39] D. Hexner, A. J. Liu, and S. R. Nagel, Phys. Rev. Lett. 121, 115501 (2018).

[40] V. I. Arnol'd, Catastrophe Theory, 3rd ed. (Springer, Berlin, 1992). 